\title{
KAM TORI AND WHISKERED INVARIANT TORI FOR NON-AUTONOMOUS SYSTEMS
}

\author{
MARTA CANADELL* AND RAFAEL DE LA LLAVE
}

\begin{abstract}
Aвstract. We consider non-autonomous dynamical systems which converge to autonomous (or periodic) systems exponentially fast in time. Such systems appear naturally as models of many physical processes affected by external pulses.

We introduce definitions of non-autonomous invariant tori and nonautonomous whiskered tori and their invariant manifolds and we prove their persistence under small perturbations, smooth dependence on parameters and several geometric properties (if the systems are Hamiltonian, the tori are Lagrangian manifolds). We note that such definitions are problematic for general time-dependent systems, but we show that they are unambiguous for systems converging exponentially fast to autonomous.

The proof of persistence relies only on a standard implicit function theorem in Banach spaces and it does not require that the rotations in the tori are Diophantine nor that the systems we consider preserve any geometric structure. We only require that the autonomous system preserves these objects. In particular, when the autonomous system is integrable, we obtain the persistence of tori with rational rotational. We also discuss fast and efficient algorithms for their computation. The method also applies to infinite dimensional systems which define a good evolution, e.g. PDE's.

When the systems considered are Hamiltonian, we show that the time dependent invariant tori are isotropic. Hence, the invariant tori of maximal dimension are Lagrangian manifolds. We also obtain that the (un)stable manifolds of whiskered tori are Lagrangian manifolds.

We also include a comparison with the more global theory developed in [BdIL11].
\end{abstract}

\section{Contents}

\section{Introduction}

Date: July 31, 2015.

2010 Mathematics Subject Classification. 37C60 34L25 37J .

Key words and phrases. Dynamical Systems, KAM Tori, time dependent.

M.C. and R.L. supported in part by NSF DMS-1500943. M.C. also supported by MTM2012-32541.

* Email: marta.canadell@math.gatech. edu, Phone: +1 (404)894-9426. 
2. Set up and basic definitions for the time dependent KAM tori 4

2.1. The case of continuous time 4

2.2. The case of maps 6

2.3. Basic definitions $\quad 7$

3. Statement of the main theorems 8

4. Proof of the main theorems 9

4.1. Preliminary definitions 9

4.2. Proof of Theorem 3.1 10

$\begin{array}{ll}\text { 4.3. Newton algorithm } & 14\end{array}$

4.4. Proof of Theorem 3.2 15

4.5. Smooth dependence on parameters 17

5. Whiskered KAM tori 17

6. Geometric properties 20

7. Non-Euclidean manifolds 22

$\begin{array}{ll}\text { Acknowledgments } & 23\end{array}$

Appendix A. Summary of the global scattering theory for time dependent classical systems 23

$\begin{array}{ll}\text { References } & 24\end{array}$

\section{INTRODUCTION}

Many physical processes consist of a dynamical system which is subject to a perturbation which is localized in time (and may be in space too). For example, the orbit of a planet may be disturbed by a passage of anther body ([ALF05, LAAF07, SHF10]) or a molecule may be disturbed by another molecule or by a laser pulse $\left(\left[\mathrm{KBJ}^{+} 07, \mathrm{BRBB} 12, \mathrm{BMH}^{+} 08, \mathrm{BUMH} 08\right.\right.$, $\mathrm{KBJ}^{+}$07]). It becomes interesting to quantify the effect of the perturbation and analyze the dynamics of these systems.

If the dynamical system was autonomous, a standard procedure to analyze its behavior is to find invariant objects that organize the dynamics. For a non-autonomous system in general, the analogous of very simple invariant objects are far from obvious. For example, there are several natural definitions of attractors that are not equivalent [KR11, Hal88, CV02, VZC13]. Nevertheless, as shown already in [BdlL11], there is a rather satisfactory theory of non-autonomous invariant objects for systems which converge (somewhat fast) to autonomous. Indeed, in [BdlL11], it is shown that if the convergence is fast enough, there are non-autonomous changes of variables that convert the system into autonomous and that they are readily computable. Hence, using these transformations, one obtains very precise (and uniquely determined under mild conditions) non-autonomous analogues of 
invariant objects. These were computed numerically in [BF12]. Other definitions of non-autonomous invariant objects using non-autonomous normal form appear in $\left[\mathrm{KBJ}^{+} 07\right]$.

The paper [BdlL11] presents a global theory of these non-autonomous invariant objects. It shows not only their existence and their properties, but also shows how they fit together and implemented numerically in [BF12]. For the sake of completeness, we have included appendix A summarizing results of [BdlL11].

The goal of this paper is to provide rather elementary proofs of the existence and properties of non-autonomous analogues of rotational tori (which are some times called non-autonomous KAM tori), as well as non-autonomous analogues of Whiskered tori - i.e. tori with motion conjugate to a rotation and with stable and unstable directions - as well as to (un)stable manifold of whiskered tori (see Section 5). In contrast with the work of [BdlL11], we deal with one of the objects at time, which leads to faster computation.

We note that the results presented here do not require either that the frequency is Diophantine nor that flow preserves a geometric structure nor that they are close to integrable. The proof is just an application of the standard Implicit Function Theorem in Banach Spaces and does not require transformation theory, which also leads to effective algorithms (see Section 4.3). The proof presented here also works for infinitely dimensional systems, including some PDE's. The only requirement is that they define a smooth evolution operator.

The main idea we will use is to derive a functional equation giving parameterizations of the objects and then show that can be solved by an elementary implicit function theorem in Banach spaces. (We will need to define appropriate Banach spaces of sequences with weights). The main input for the theory developed here is the existence of analogous objects invariant for the autonomous systems. Of course, these invariant objects in the autonomous systems are readily obtainable if the autonomous system is integrable, but we do not assume that our systems are close to integrable. We could take as input numerically computed objects. In this paper, we assume that invariant objects for the autonomous systems are known. We point out the formalism used here matches very well the formalism of KAM theory for autonomous systems developed in [dlLGJV05, FdlLS09a, FdlLS09b]. Numerical aspects of the computation in autonomous systems appear in [HdlLS12, HdlLS13].

For the sake of simplicity, first we will present proofs for the case of mappings and, then, we will present the case of time dependent differential equations. We will show that the result for maps imply corresponding 
results for differential equations. But the results for maps have other applications: they imply results for perturbations of time-periodic differential equations; some evolutionary PDE's admit smooth evolution operators, even if the vector field generating them is an unbounded - hence discontinuous - operator.

The proof presented in this paper is not based on transformation theory, but rather using parameterizations of objects, so that a numerical implementation would need only to deal with functions of the objects and not with functions on the dimensions of the phase space. This alleviates significantly the "curse of dimensionality". We postpone the detailed discussion of numerical implementations for a future work, but we point out that there are very efficient Newton methods, see Section 4.3.

We note that a theory of invariant tori for perturbations of quadratic systems, has been developed in [FW14]. This theory is based in transformation theory and requires that the system preserves a geometric structure. The results are particular cases of the results of this paper.

\section{Set UP AND BASIC DEFINITIONS FOR THE TIME DEPENDENT KAM TORI}

In this section we present and motivate the definition of the time dependent KAM tori both for maps and for differential equations. In subsequent sections, we will present similar treatments for other invariant objects (whiskered tori and their manifolds). It seems clear that variations on the approach can be used to extend other invariant objects to the non-autonomous context. In this section, we will present only the formal aspects. To formulate the hypothesis of the theorems, we will need to make precise the sense in which convergence takes place and this will require to introduce norms, etc. This rigorous treatment will be done in Section 3 .

2.1. The case of continuous time. We will consider time dependent differential equations. For typographical convenience, we denote the time dependence of the vector field by a subindex. Hence, we will consider a differential equation for the trajectories $x(t)$ as follows

$$
\dot{x}(t)=\frac{\mathrm{d}}{\mathrm{d} t} x(t)=X_{t}(x(t)),
$$

where $X_{t}$ is a time dependent vector field in the phase space $M$.

We denote by $V_{t_{0}}^{t}$ the time advance diffeomorphism. That is, if $x(t)$ is a trajectory of (1), we have

$$
x(t)=V_{t_{0}}^{t}\left(x\left(t_{0}\right)\right)
$$

which represents the evolution of a trajectory in time (the flow). Clearly, for all $t_{2}, t_{1}, t_{0}$ :

$$
V_{t_{1}}^{t_{2}} \circ V_{t_{0}}^{t_{1}}=V_{t_{0}}^{t_{2}} ; \quad V_{t}^{t}=\mathrm{Id}
$$


We will similarly denote by $U_{t}$ the time advance of the autonomous differential equation $X_{\infty}$. Since $X_{\infty}$ is autonomous, the time advance depends only on the difference between the initial and the final time,

$$
U_{t} \circ U_{s}=U_{t+s} ; U_{0}=\mathrm{Id} \text {. }
$$

In the following definitions, we will introduce the concepts we will be using.

Definition 2.1. We say that a family of embeddings $K_{t}: \mathbb{T}^{d} \rightarrow M$ is a non-autonomous $K A M$ torus when it satisfies the invariance equation

$$
X_{t}\left(K_{t}(\theta)\right)=\partial_{t} K_{t}(\theta)+\left(\omega \cdot \partial_{\theta}\right) K_{t}(\theta),
$$

for any $\omega \in \mathbb{R}^{d}$.

Note that, for the sake of brevity, we are using the name non-autonomous $K A M$ torus when it would have been more precise to talk about the embeddings giving a KAM torus. This does not cause confusion.

In the autonomous case, Definition 2.1 is the same definition used in [dlLGJV05]. In ergodic theory for the autonomous case, this means that the rotation is a factor of the full dynamics.

Note that if (2) is satisfied, we will have a corresponding equation in terms of the time advance diffeomorphism

$$
V_{t_{0}}^{t}\left(K_{t_{0}}(\theta)\right)=K_{t}\left(\theta+\omega\left(t-t_{0}\right)\right)
$$

Conversely, (3) implies (2) for ordinary differential equations. See, however Remark 2.4.

The reason we call (2) and (3) an invariance equation is that

$$
V_{t_{0}}^{t}\left(K_{t_{0}}\left(\mathbb{T}^{d}\right)\right)=K_{t}\left(\mathbb{T}^{d}\right) .
$$

That is, the manifolds $\mathcal{T}_{t}=K_{t}\left(\mathbb{T}^{d}\right)$ are transported into each other by the dynamics. If we perform the standard construction [Hal80] of making the system autonomous by considering an extended phase space given by $(x, t)$ and the extended (autonomous) dynamics

$$
(x, t)^{\prime}=\left(X_{t}(x), 1\right),
$$

then, (3) is just the invariance in the extended system.

We will supplement the invariance condition (2) or (3) with the asymptotic condition

$$
K_{t} \rightarrow K_{\infty} .
$$

In particular, notice that if condition (4) is satisfied, we will have

$$
X_{\infty} \circ K_{\infty}=\left(\omega \cdot \partial_{\theta}\right) K_{\infty}(\theta) .
$$

So that, $K_{\infty}$ will give a parameterization of a KAM torus for $X_{\infty}$. 
Remark 2.2. Note that the existence of solution (2) (or (3)) is trivial. If we chose any initial embedding $K_{0}$ and then take the right hand side of (3) as a definition of $K_{t}$, we obtain a solution. We emphasize that the point of the theory developed here is that the tori produced are well behaved for long time and, hence provide useful landmarks. The tori obtained by propagating an initial torus will, in general, become more complicated objects very fast. The tori we consider here are selected by the fact that after long time, they remain smooth and have a well defined limit.

In a topological language: given any initial torus, the evolution - being a homeomorphism- leaves it a torus. So, it is trivial to get tori that evolve in time. Of course, we can always reparameterize to flow so that it is a rotation. The thing which is non-trivial (indeed determines the torus uniquely) is that the long term behavior leaves the torus in good shape and that it has the right rotation. Most tori chosen will become rather wild objects.

Remark 2.3. Notice that solutions $K_{\infty}$ of (5) are not unique. Indeed, if $K_{\infty}$ solves equation (5), we have that for every $\sigma \in \mathbb{R}^{d}$ :

$$
U_{t} \circ\left(K_{\infty} \circ T_{\sigma}\right)=K_{\infty} \circ T_{t \omega} \circ T_{\sigma}=\left(K_{\infty} \circ T_{\sigma}\right) \circ T_{\omega},
$$

where $T_{\omega}(\theta)=\theta+\omega$. So that $K_{\infty} \circ T_{\sigma}$ also satisfies (5). This ambiguity corresponds to the choice of the change of origin in the reference torus, but the geometric object is the same. Nevertheless, we will show that, once we choose a parameterization of the autonomous invariant torus, the nonautonomous invariant torus is uniquely determined. Of course, if rather than choosing $K_{\infty}$ we chose $K_{\infty} \circ T_{\sigma}$, the corresponding time dependent invariant tori would be $K_{n} \circ T_{\sigma}$.

Remark 2.4. The precise conditions will be given later, but we note that the main assumption is that we can define the evolution. In PDE's one can sometime define good evolution operators even if the "vector field" $X$ is an unbounded operator. Of course, if $X$ is a smooth vector field - even in infinite dimensions - we obtain a nice evolution. Hence the formulation of the problem (3) is more general in infinite dimensions than (2).

2.2. The case of maps. We will consider $f_{n}$, a sequence of diffeomorphisms:

$$
f_{n} \rightarrow f_{\infty}
$$

where the index $n$ denotes time, so that an orbit is a sequence of points $\left\{x_{n}\right\}_{n}$ such that

$$
x_{n+1}=f_{n}\left(x_{n}\right) .
$$

Remark 2.5. If $X_{t}$ is a time dependent vector field, we can consider $f_{n}$ to be a map that gives the positions at time $n+1$ as a function of positions at time $n$, see Section 2.1. 
Definition 2.6. We say that the time dependent KAM tori (non-autonomous KAM tori) are a sequence of embeddings $K_{n}: \mathbb{T}^{d} \rightarrow M$ such that satisfies the invariance equation:

$$
f_{n} \circ K_{n}(\theta)=K_{n+1}(\theta+\omega)
$$

for any $\omega \in \mathbb{R}^{d}$.

In analogy with the case of flows, we will supplement the invariance equation (6) with the asymptotic condition

$$
K_{n} \rightarrow K_{\infty}
$$

Notice that if we have condition (7) satisfied, we will have

$$
f_{\infty} \circ K_{\infty}(\theta)=K_{\infty}(\theta+\omega) .
$$

So that, $K_{\infty}$ will give a parameterization of a (autonomous) KAM torus for $f_{\infty}$.

Again, we note that (6) indicates that the range of $K_{n}$ is transported by $f_{n}$ into the range of $K_{n+1}$ and that these ranges are uniformly smooth. Notice that (8) is the formulation used in [dlLGJV05]. This justifies that we call the solutions KAM tori.

2.3. Basic definitions. For the sake of simplicity, we will discuss first in detail the case when the phase space $M$ is a Euclidean manifold so that we can add vectors and consider mappings on the manifold as a elements in a Banach space of mappings. Of course, it is standard to use the exponential mapping to show that the space of diffeomorphisms is a Banach manifold. See Section 7.

In order to prove the results of the theorems, we need to define appropriate norms. The following definitions are standard, see [Die69, Sch69].

We consider the Banach space of $r$-continuously differentiable functions $f: M \rightarrow M$ endowed with the norm

$$
\|f\|_{C^{r}}=\left\|f^{(r)}\right\|_{\infty}=\max _{z \in M}\left|f^{(r)}(z)\right|
$$

where the superindex $(r)$ means the $r$-derivative of the function $f$. Analogously, we consider the Banach space of $r$-continuously differentiable embeddings $K: \mathbb{T}^{d} \rightarrow M$ endowed with the norm

$$
\|K\|_{C^{r}}=\left\|K^{(r)}\right\|_{\infty}=\max _{\theta \in M}\left|K^{(r)}(\theta)\right| .
$$

Remark 2.7. Note that these definitions make sense even when $M$ is an infinite dimensional manifold. In that case, we take the convention that the derivatives means strong derivatives, also called Fréchet derivatives. 


\section{Statement of the Main theorems}

The main theorems on KAM tori for maps and flows respectively are formulated below.

Theorem 3.1 (Case of maps). Let $M$ be a (Euclidean) manifold. Let $f_{n}$ : $M \rightarrow M$ be a sequence of diffeomorphisms such that $\left\|f_{n}-f_{\infty}\right\|_{C^{r+2}} \leq C_{1} \lambda^{n}$, for some $\lambda \leq 1$ and $C_{1}>0$. Assume that $f_{\infty}$ has a KAM torus, that is, there exists an embedding $K_{\infty}: \mathbb{T}^{d} \rightarrow M$ and $\omega \in \mathbb{R}^{d}$ such that

$$
f_{\infty} \circ K_{\infty}(\theta)=K_{\infty}(\theta+\omega) \text {. }
$$

Assume that for some $\mu>0, C>0$, we have

(I) $\left\|\mathrm{D} f_{\infty} \circ K_{\infty}(\theta+n \omega) \cdots \mathrm{D} f_{\infty} \circ K_{\infty}(\theta)\right\|_{C^{0}} \leq \mu^{n} C$,

(II) $\mu^{r} \lambda<1$,

(III) $C_{1}$ sufficiently small.

Then, there exists a sequence of embeddings $K_{n}: \mathbb{T}^{d} \rightarrow M$ satisfying (6) and

$$
\left\|K_{n}-K_{\infty}\right\|_{C^{r}} \leq C_{*} \lambda^{n} .
$$

Furthermore, we can take $C_{*}=L C_{1}$ where $L$ is a constant depending only on the sequence $f_{n}$.

The sequence $K_{n}$ satisfying (6) and (10) is, with $C_{*} \leq C_{+}$, (where $C_{+}$ depends only on $f_{n}$, unique.

Theorem 3.2 (Case of Flows). Let $M$ be a manifold. Let $\dot{x}(t)=X_{t}(x(t))$ be a differential equation that

$$
\left\|X_{t}-X_{\infty}\right\|_{C^{r+1}} \leq C_{2} \lambda^{t}
$$

for some $\lambda \leq 1$ and $C_{2}>0$.

Assume that $X_{\infty}$ has a KAM torus, that is, there exists an embedding $K_{\infty}: \mathbb{T}^{d} \rightarrow M$ and $\omega \in \mathbb{R}^{d}$ such that

$$
X_{\infty} \circ K_{\infty}(\theta)=\omega \frac{\partial}{\partial \theta} K_{\infty}(\theta) .
$$

Assume also that $V_{t_{0}}^{t}$ defines the time advance diffeomorphism of this differential equation, such that

$$
V_{t_{0}}^{t}\left(K_{t_{0}}(\theta)\right)=K_{t}\left(\theta+\omega\left(t-t_{0}\right)\right)
$$

with $U_{t}$ being the time advance of $X_{\infty}$. Assume that for some $\mu>0, C>0$, we have

(I) $\left\|\mathrm{D} U_{n} \circ K_{\infty}(\theta) \cdots \mathrm{D} U_{0} K_{\infty}(\theta)\right\|_{L^{\infty}\left(\mathbb{T}^{d}\right)} \leq \mu^{n} C$,

(II) $\mu^{r} \lambda<1$,

(III) $C_{2}$ sufficiently small. 
Then, there exists a continuous family of embeddings $K_{t}: \mathbb{T}^{d} \rightarrow M$ satisfying (12) and

$$
\left\|K_{t}-K_{\infty}\right\|_{C^{r}} \leq C_{* *} \lambda^{t} .
$$

Furthermore, we can take $C_{* *}=L C_{2}$, where $L$ is a constant depending only on $X_{t}$.

The sequence $K_{n}$ satisfying (12) and (13) for some $C_{* *} \leq C_{+}$(where $C_{+}$ depends only depending only on $X_{t}$ ) is unique.

Remark 3.3. Note that in Theorem 3.1 (respectively in Theorem 3.2) we are not assuming that $\omega$ is Diophantine nor that $f_{\infty}$ (respectively $X_{\infty}$ ) preserves any geometric structure and much less that $f_{\infty}$ (respectively $X_{\infty}$ ) is integrable. Of course, if $f_{\infty}$ (respectively $X_{\infty}$ ) is integrable, we can take $\omega$ to be any vector, even rational. Hence, Theorem 3.1 and Theorem 3.2 give the persistence of all frequencies under time dependent perturbation.

Remark 3.4. If $f_{\infty}$ (respectively $X_{\infty}$ ) preserves symplectic structure and the torus is Lagrangian, we can take $\mu$ to be any number bigger than 1 .

Remark 3.5. In the case that $f_{\infty}$ and $f_{n}$ are symplectic, we will obtain more properties for the tori, see Section 6.

Remark 3.6. In the case that the systems are analytic, one can obtain analyticy results too. It suffices to note that if we consider the systems defined in a complex neighborhood and the $f_{n}$ are differentiable (in the complex sense), the proof presented here obtains that the $K_{n}$ are also differentiable (in the complex sense). Alternatively, define spaces of analytic embeddings defined in a complex extensions of the tori and follow the $C^{0}$ argument here.

We can think of Theorems 3.1 and 3.2 as a functional that giving a sequence $f_{n}$ (respectively a time dependent vector field $X_{t}$ ) produces a sequence of $K_{n}$. In section 4.5, we will investigate the properties of this functional and we will establish that when the sequences $f_{n}$ and $K_{n}$ are giving appropriate topologies, this functional is differentiable and obtain expressions for its derivatives. This is a very natural question since in many applications, the $f_{n}$ depends on parameters and it is useful to quantify the effect of changing the parameters on the tori.

\section{Proof of The MaIn theorems}

4.1. Preliminary definitions. We denote by $\underline{f}_{\infty}$ and $\underline{K}_{\infty}$ the constant sequences whose elements are $f_{\infty}$ and $K_{\infty}$, respectively.

We define the following affine spaces of sequences of diffeomorphisms on $M$ and of embeddings $\mathbb{T}^{d} \rightarrow M$. 
Definition 4.1 (Spaces of sequences of maps). We define a space of sequences of diffeomorphisms on $M$ by

$$
\mathcal{F}_{\lambda, r}=\left\{\left\{f_{n}\right\}_{n=0}^{\infty} \mid\left\|f_{n}-f_{\infty}\right\|_{C^{r}} \leq C \lambda^{n}\right\} .
$$

Furthermore, we define a space of sequences of embeddings on $\mathbb{T}^{d} \rightarrow M$ as

$$
\mathcal{K}_{\lambda, r}=\left\{\left\{K_{n}\right\}_{n=0}^{\infty} \mid\left\|K_{n}-K_{\infty}\right\|_{C^{r}} \leq C \lambda^{n}\right\} .
$$

Of course, we define for the sequences $f, \tilde{f} \in \mathcal{F}_{\lambda, r}$ and $\underline{K}, \tilde{K} \in \mathcal{K}_{\lambda, r}$

$$
\begin{aligned}
\|\underline{f}-\tilde{f}\|_{\lambda, r} & =\sup _{n}\left\|f_{n}-f_{\infty}\right\|_{C^{r}} \lambda^{-n}, \\
\|\underline{K}-\tilde{K}\|_{\lambda, r} & =\sup _{n}\left\|K_{n}-K_{\infty}\right\|_{C^{r}} \lambda^{-n} .
\end{aligned}
$$

Note that $\mathcal{F}_{\lambda, r}$ and $\mathcal{K}_{\lambda, r}$ are affine spaces modeled on the Banach spaces

$$
\begin{aligned}
& \mathcal{F}_{\lambda, r}^{0}=\left\{\left\{\hat{f}_{n}\right\}_{n=0}^{\infty} \mid\left\|\hat{f}_{n}\right\|_{C^{r}} \leq C \lambda^{n}, \text { with }\left\|\hat{f}_{n}\right\|_{\lambda, r}=\sup \left\|\hat{f}_{n}\right\|_{C^{r}} \lambda^{-n}\right\}, \\
& \mathcal{K}_{\lambda, r}^{0}=\left\{\left\{\hat{K}_{n}\right\}_{n=0}^{\infty} \mid\left\|\hat{K}_{n}\right\|_{C^{r}} \leq C \lambda^{n}, \text { with }\left\|\hat{K}_{n}\right\|_{\lambda, r}=\sup \left\|\hat{K}_{n}\right\|_{C^{r}} \lambda^{-n}\right\},
\end{aligned}
$$

respectively.

For the case of flows, we will assume that

$$
\left\|X_{t}-X_{\infty}\right\|_{C^{r+1}} \leq C_{2} \lambda^{t}
$$

for some $\lambda<1$ and $C_{2}>2$, where $\|\cdot\|_{C^{r}}$ is defined in Section 2.3.

We note that, using the theory of smooth dependence of solutions of differential equations [Hal80, Har02] this implies

$$
\left\|V_{t_{0}}^{t_{0}+a}-U_{a}\right\|_{C^{r}} \leq c_{r} \lambda^{-t_{0}}
$$

for all $t \in \mathbb{N}$ and $|a| \leq 2$.

We note that (14) is the only thing that we will need to carry out the theory. One can get them in PDE's using semi-group theory for some evolution equations. Since this requires considerations specific to the model considered, we will postpone the discussion.

4.2. Proof of Theorem 3.1. We define the functional $\mathcal{J}$ as

$$
\mathcal{J}: \mathcal{F}_{\lambda, r+2} \times \mathcal{K}_{\lambda, r} \rightarrow \mathcal{K}_{\lambda, r}^{0}
$$

by

$$
\mathcal{J}(\underline{f}, \underline{K})_{n}=f_{n} \circ K_{n}-K_{n+1} \circ T_{\omega} .
$$

Note that the invariance of $K_{\infty}$ under $f_{\infty}$ given in (9) is written as

$$
\mathcal{J}\left(\underline{f}_{\infty}, \underline{K}_{\infty}\right)=\underline{f}_{\infty} \circ \underline{K}_{\infty}-\underline{K}_{\infty} \circ T_{\omega}=0 .
$$


We want to use the Implicit Function Theorem in Banach spaces to conclude that if $f$ is close to $\underline{f}_{\infty}$ in the $\mathcal{F}_{\lambda, r+2}$ topology, we get a locally unique $\underline{K} \in \mathcal{K}_{\lambda, r}$ such that

$$
\mathcal{J}(\underline{f}, \underline{K})=0,
$$

which clearly implies Theorem 3.1. Using the Implicit Function Theorem in Banach spaces [Die69, Sch69], the proof of Theorem 3.1 is immediate from the following two lemmas 4.2 and 4.3.

Lemma 4.2 (Differentiability). The functional $\mathcal{J}$ defined by

$$
\mathcal{J}: \mathcal{F}_{\lambda, r+l+1} \times \mathcal{K}_{\lambda, r} \rightarrow \mathcal{K}_{\lambda, r}^{0}
$$

is a $C^{l}$ function.

Furthermore, assume that $0<\lambda<1, r \in \mathbb{N}, l \in \mathbb{N}$ and $l \geq 1$, then

$$
\left(\mathrm{D}_{2} \mathcal{J}(\underline{f}, \underline{K}) \Delta\right)_{n}:=\mathrm{D} f_{n} \circ K_{n} \Delta_{n}-\Delta_{n+1} \circ T_{\omega}
$$

and

$$
\mathrm{D}_{2} \mathcal{J}: \mathcal{F}_{\lambda, r} \times \mathcal{K}_{\lambda, r} \rightarrow L\left(\mathcal{K}_{\lambda, r}^{0}, \mathcal{K}_{\lambda, r}^{0}\right)
$$

is a Lipschitz mapping.

Lemma 4.3 (Invertibility). With the notation above, the derivative

$$
\mathrm{D}_{2} \mathcal{J}\left(\underline{f}_{\infty}, \underline{K}_{\infty}\right): \mathcal{F}_{\lambda, r}^{0} \times \mathcal{K}_{\lambda, r}^{0} \rightarrow \mathcal{K}_{\lambda, r}^{0}
$$

is boundedly invertible under the rate conditions (I) and (II) of Theorem 3.1.

Remark 4.4. The regularity assumption on $\underline{f}_{\infty}$ in Lemma 4.2 is too conservative. It suffices to assume $\underline{f}_{\infty} \in \mathcal{F}_{\lambda, r+l}$ as well as some assumption on the uniform continuity of $\mathrm{D}^{l+r} f_{n}$. Of course, this is implied by our assumptions. We have selected some properties that imply the desired result but which does not need any new notation. A very detailed study of regularity of composition operator appears in [dlLO99, Irw72].

Lemma 4.2. By the Fundamental Theorem of Calculus, given a sequence $\left\{K_{n}\right\}$ in the affine space $\mathcal{K}_{\lambda, r}$ and a sequence $\left\{\Delta_{n}\right\}$ in the linear space $\mathcal{K}_{\lambda, r}^{0}$, we note that $\left\{K_{n}+\Delta_{n}\right\}$ is in the affine space $\mathcal{K}_{\lambda, r}$, and we have:

$$
\begin{aligned}
f_{n} \circ\left(K_{n}+\Delta_{n}\right) & -f_{n} \circ K_{n}-\mathrm{D} f_{n} \circ K_{n} \Delta_{n}= \\
& \left.=\int_{0}^{1}\left(\mathrm{D} f_{n} \circ\left(K_{n}+s \Delta_{n}\right) \Delta_{n}-\mathrm{D} f_{n} \circ K_{n} \Delta_{n}\right)\right) \mathrm{d} s \\
& =\int_{0}^{1}\left(\int_{0}^{s} \mathrm{D}^{2} f_{n} \circ\left(K_{n}+s w \Delta_{n}\right) \Delta_{n}^{\otimes 2} \mathrm{~d} w\right) \mathrm{d} s .
\end{aligned}
$$


Therefore, we can bound:

$$
\begin{aligned}
\| f_{n} \circ\left(K_{n}+\Delta_{n}\right)-f_{n} \circ K_{n} & -\mathrm{D} f_{n} \circ K_{n} \Delta_{n} \|_{C^{r}} \leq \\
& \leq C\left\|\mathrm{D}^{2} f_{n}\right\|_{C^{r}}\left(1+\left\|K_{n}\right\|_{C^{r}}\left\|\Delta_{n}\right\|_{C^{r}}\right)\left\|\Delta_{n}\right\|_{C^{r}}^{2} \\
& \leq C\left\|f_{n}\right\|_{C^{r+2}}\left(1+\left\|K_{n}\right\|_{C^{r}}\left\|\Delta_{n}\right\|_{C^{r}}\right) \lambda^{n}\|\underline{\Delta}\|_{\lambda, r}^{2} \\
& \leq C \lambda^{n+1}\|\underline{\Delta}\|_{\lambda, r}^{2},
\end{aligned}
$$

for some constant $C>0$ depending only of $f$. And, of course,

$$
\left(K_{n+1}+\Delta_{n+1}\right) \circ T_{\omega}=K_{n+1} \circ T_{\omega}+\Delta_{n+1} \circ T_{\omega} .
$$

This shows that $\mathcal{J}$ is also differentiable in the second argument $K$. The differentiability of $\mathcal{J}$ with respect to $f_{n}$ is clear because $\mathcal{J}$ is linear in $f_{n}$. We also obtain the expression of the partial derivatives of $\mathcal{J}$ given in (15).

The same argument also gives that the derivative of $\mathcal{J}$ is Lipschitz with respect to the argument, since from the following expression

$$
\mathrm{D} f_{n} \circ\left(K_{n}+\Delta_{n}\right)-\mathrm{D} f_{n} \circ K_{n}=\int_{0}^{1}\left(\mathrm{D}^{2} f_{n}\left(K_{n}+s \Delta_{n}\right) \Delta_{n}\right) \mathrm{d} s,
$$

we can bound the left hand side by

$$
\left\|\mathrm{D} f_{n} \circ\left(K_{n}+\Delta_{n}\right)-\mathrm{D} f_{n} \circ K_{n}\right\|_{C^{r}} \leq C\left\|\Delta_{n}\right\|_{C^{r}} \leq C\|\underline{\Delta}\|_{\lambda, r} \lambda^{n},
$$

for some constant $C>0$, which again depends only on $f$.

This gives the case $l=1$ of Lemma 4.2, which is the only one we need in the applications to existence (but higher derivative are useful to obtain dependence on parameters). To get higher derivatives, we just observe that we can apply the case already proved for $\mathrm{D} f_{n}$ in place of $f_{n}$. This gives the case $l=2$, and so on. This completes the proof of Lemma 4.2.

Lemma 4.3. What we need to show is that given $\underline{\eta} \in \mathcal{K}_{\lambda, r}^{0}$, we can find a unique $\Delta \in \mathcal{K}_{\lambda, r}^{0}$ solving

$$
\mathrm{D} f_{\infty} \circ K_{\infty} \Delta_{n}-\Delta_{n+1} \circ T_{\omega}=\eta_{n}
$$

and that

$$
\|\Delta\|_{\lambda, r} \leq C\|\underline{\eta}\|_{\lambda, r}
$$

We rewrite equation (16) in components as:

$$
\Delta_{n}=M \eta_{n}+M \Delta_{n+1} \circ T_{\omega},
$$

where $M=\left(\mathrm{D} f_{\infty} \circ K_{\infty}\right)^{-1}$. Applying equation (16) repeatedly we are led to the fact that (16) is equivalent to:

$$
\begin{aligned}
\Delta_{n}= & \sum_{j=0}^{L} M \circ T_{\omega}^{j} \cdots M \eta_{n+j} \circ T_{\omega}^{j} \\
& +M \circ T_{\omega}^{L+1} \cdots M \Delta_{n+L+1} \circ T_{\omega}^{L+1} .
\end{aligned}
$$


Formally, Equation (17) suggests that we should have

$$
\Delta_{n}(\theta)=\sum_{j=0}^{\infty} M \circ T_{\omega}^{j}(\theta) \cdots M(\theta) \eta_{n+j} \circ T_{\omega}^{j}(\theta) .
$$

Let us see that indeed equation (18) is well defined and gives the unique solution of equation (16). This will rely on estimating the $C^{r}$ norm of the general term in (17), (18) and the remainder in (17).

We need to recall and rewrite the assumption (I) of the theorem as:

$$
\left|M \circ T_{\omega}^{j}(\theta) \cdots M(\theta)\right| \leq c_{0} \mu^{j},
$$

for some $c_{0}>0$. We first estimate $\partial_{\theta}^{r}\left(M \circ T_{\omega}^{j}(\theta) \cdots M(\theta)\right)$. Notice that the derivatives will be a product of $T_{j}^{r}$ terms, each which has at most $F_{j}^{r}$ terms. Using the product rule, we obtain the recursion in $r$ :

$$
\begin{aligned}
& T_{j}^{r+1} \leq T_{j}^{r} F_{j}^{r}, \\
& F_{j}^{r+1} \leq F_{j+1}^{r} .
\end{aligned}
$$

We also have the initial conditions $(r=0)$ for the recursions (19) and (20)

$$
F_{j}^{0}=j, \quad T_{j}^{0}=1 .
$$

Using the recursion (19)-(20) above and the initial conditions, we have

$$
\begin{aligned}
& T_{j}^{r} \leq j(j+1) \cdots(j+r), \\
& F_{j}^{r} \leq j+r .
\end{aligned}
$$

Furthermore, we observe that all the terms in the expression for $\partial_{\theta}^{r}(M \circ$ $\left.T_{\omega}^{j}(\theta) \cdots M(\theta)\right)$ are products of strings of $M \circ T_{\omega}^{l+m} \cdots M \circ T_{\omega}^{l}$, whose length add up to $j-r$, separated by derivatives of $M$ of order not more than $r$. Hence, we obtain:

$$
\left\|\partial_{\theta}^{r}\left(M \circ T_{\omega}^{j} \cdots M(\theta)\right)\right\|_{C^{r}} \leq c_{0}^{r}\|M\|_{C^{r}}^{r} j(j+1) \cdots(j+r) \mu^{r j} .
$$

From the bounds of Equation (21) and the definition of $\mathcal{K}_{\lambda, r}^{0}$, we obtain that, if there is a solution $\Delta$ of Equation (17) in $\mathcal{K}_{\lambda, r}^{0}$, the last term goes to 0 in $C^{r}$. Hence, we should have

$$
\begin{aligned}
\Delta_{n}= & \lim _{L \rightarrow \infty} \sum_{j=0}^{L} M \circ T_{\omega}^{j} \cdots M \eta_{n+j} \circ T_{\omega}^{j} \\
& +M \circ T_{\omega}^{L+1} \cdots M \Delta_{n+L+1} \circ T_{\omega}^{L+1} .
\end{aligned}
$$

where the limit is understood in $C^{r}$. So, we know that the solution $\Delta_{n}$ given in (18) is the only possible solution.

Now that we know that Equation (18) is the only possible solution, we investigate its properties and show that indeed is a solution and satisfies the 
desired bounds. Using Equation (21), we can bound the general term of Equation (18) by:

$$
\begin{aligned}
\left\|M \circ T_{\omega}^{j} \cdots M \eta_{n+j} \circ T_{\omega}^{j}\right\|_{C^{r}} & \leq\left\|M \circ T_{\omega}^{j} \cdots M\right\|_{C^{r}}\left\|\eta_{n+j}\right\|_{C^{r}} \\
& \leq c_{0}^{r}\|M\|_{C^{r}}^{r} j(j+1) \cdots(j+r) \mu^{r j} \lambda^{n+j}\|\eta\|_{\lambda, r} \\
& =\lambda^{n}\|\eta\|_{\lambda, r} c_{0}^{r}\|M\|_{C^{r}}^{r} \frac{(j+r) !}{j-1}\left(\mu^{r} \lambda\right)^{j} .
\end{aligned}
$$

Therefore, the series of Equation (18) converges uniformly, since by assumption (II) of Theorem 3.1 we have $\mu^{r} \lambda<1$. Hence, it is not difficult to justify it is a solution of (16) by rearranging terms of series. We finally observe that

$$
\left\|\Delta_{n}\right\|_{C^{r}} \leq \lim _{L \rightarrow \infty} \sum_{j=0}^{L} \lambda^{n}\|\eta\|_{\lambda, r} c_{0}^{r}\|M\|_{C^{r}}^{r} \frac{(j+r) !}{j-1}\left(\mu^{r} \lambda\right)^{j} \leq C \lambda^{n}\|\eta\|_{\lambda, r} \leq C_{*} \lambda^{n},
$$

where the constant $C_{*}>0$ depends only on the sequence $f_{n}$.

Remark 4.5. In the case that $f_{\infty}$ is an integrable mapping and $K_{\infty}$ is the parameterization of a KAM torus, then we have

$$
M=\left(\begin{array}{cc}
\mathrm{Id} & A \\
0 & \mathrm{Id}
\end{array}\right),
$$

which is constant. So, the norm of $\left\|M \circ T_{\omega}^{j} \cdots M\right\|_{C^{r}}$ grows linearly in $j$.

Remark 4.6. In the case that $K_{\infty}$ is a non-twist torus (i.e., a torus for which the torsion vanishes), we have

$$
M=\left(\begin{array}{cc}
\text { Id } & 0 \\
0 & \text { Id }
\end{array}\right)
$$

and the norm $\left\|M \circ T_{\omega}^{j} \cdots M\right\|_{C^{r}}$ do not grow. Note that this shows that nontwist tori are more stable under non stationary perturbations, which gives a rigorous meaning to the observation that non-twist KAM tori are "more robust". See [MEdCCR10, dCNGM96, Mor00, Eas91]. We also note that the models in [FW14], satisfy (22) since the unperturbed systems is a harmonic oscillator.

4.3. Newton algorithm. Note that the proof of Theorem 3.1 can be easily turned into a Newton-like method, which is quadratically convergent.

If we define $\mathcal{F}(f, K)=E$, where $E$ represents the error of the invariance equation, the Newton method just consists in setting $K$ to $K+\Delta$, where $\Delta$ satisfies

$$
\mathrm{D}_{2} \mathcal{F}(f, K) \Delta=-E .
$$

According to Lemma 4.2, for the non-autonomous case this is

$$
\mathrm{D} f_{n} \circ K_{n} \Delta_{n}-\Delta_{n+1} \circ T_{\omega}=-E_{n+1} \text {. }
$$


Proceeding as in Lemma 4.3 we obtain that the solution of (23) is given by

$$
\Delta_{n}=\sum_{j=0}^{\infty} M \circ T_{\omega}^{j} \cdots M E_{n+j} \circ T_{\omega}^{j} .
$$

We note that this is quite practical for computer implementations, especially because there are fast algorithms to compute the sums in (24). See [HdlLS12]. The Newton method, of course, has a quadratic convergence, so that a few steps can produce very accurate results. Note that the Newton method does not require to store (and much less invert) a matrix of the dimension the variables of discretization. Note also that the $K_{n}$ have the same number of variables than the tori. Since the cost of manipulating functions increases exponentially fast with the dimension (the curse of dimensionality), this method is much more efficient than the methods of transformation theory that require using functions of the dimension of the phase space.

4.4. Proof of Theorem 3.2. In this section, we present a proof of Theorem 3.2, the persistence result for flows by reducing it to Theorem 3.1. A direct proof is also possible, but reducing it to the case of maps is advantageous in cases such as dissipative or dispersive PDE's where the evolution is much nicer than the generator of the evolution.

Our goal is thus, to produce solutions of (11) using the result of Theorem 3.1 .

We fix an invariant torus $K_{\infty}$. We note that for any $a \in \mathbb{R}, K_{\infty} \circ T_{a \omega}$ is also a parameterization of an invariant torus.

Clearly, if we restrict our attention only to integer times, we can apply the result for maps. If we fix $a \in[0,2]$ we can define $f_{n}^{a}=V_{n+a}^{n+a+1}$ for all integers $n$ and apply Theorem 3.1 to obtain a solution $K_{n}^{a}$ that satisfies

$$
f_{n}^{a} \circ K_{n}^{a}=K_{n+1}^{a} \circ T_{\omega}
$$

as well as the asymptotic condition:

$$
K_{n}^{a} \rightarrow K_{\infty} \circ T_{a \omega}
$$

Our next goal is to show that family $K_{n}^{a}$ of solutions of the discrete equation is in fact a continuous family that satisfies (11) for all time (not necessarily $n \in \mathbb{N}$ ). The key to do that will be to take advantage of the uniqueness properties of Theorem 3.1 to see that indeed they satisfy the invariance equation (6) for all time.

By applying a time advance $V_{n+a+1}^{n+a+b+1}$ on the right to both sides of invariance equation (25), we obtain:

$$
V_{n+a+1}^{n+a+b+1} \circ V_{n+a}^{n+a+1} \circ K_{n}^{a}=V_{n+a+1}^{n+a+b+1} K_{n+1}^{a} \circ T_{\omega} .
$$


Using the properties of the evolution, we can rewrite the previous expression as

$$
V_{n+a+b}^{n+a+b+1} \circ\left[V_{n+a}^{n+a+b} \circ K_{n}^{a}\right]=\left[V_{n+a+1}^{n+a+b+1} \circ K_{n+1}^{a}\right] \circ T_{\omega} .
$$

In other words, $V_{n+a}^{n+a+b} \circ K_{n}^{a}$ satisfies the same invariance equation as $K_{n}^{a+b}$ (just apply (25) using $a=a+b$ ).

Moreover, since as $n \rightarrow \infty$ we have the asymptotic conditions:

$$
\begin{aligned}
K_{n}^{a} & \rightarrow K_{\infty} \circ T_{a \omega}, \\
V_{n+a}^{n+a+b} & \rightarrow U_{b},
\end{aligned}
$$

and as $U_{b} \circ K_{\infty}=K_{\infty} \circ T_{\omega b}$, then, we obtain

$$
V_{n+a}^{n+a+b} \circ K_{n}^{a} \rightarrow U_{b} \circ K_{\infty} \circ T_{a \omega}=K_{\infty} \circ T_{(a+b) \omega} .
$$

Therefore, $V_{n+a}^{n+a+b} \circ K_{n}^{a}$ satisfies the same discrete time invariance equation and the same asymptotics as $K_{n}^{a+b}$. Hence, by the local uniqueness, established in Theorem 3.1, we obtain that

$$
V_{n+a}^{n+a+b} \circ K_{n}^{a}=K_{n}^{a+b} .
$$

We also observe that if take $a=1$ we have that

$$
f_{n+1}^{1} \circ K_{n}^{1}=V_{n+1}^{n+2} \circ K_{n}^{1}=K_{n+1}^{1} \circ T_{\omega},
$$

and that $K_{n}^{1} \rightarrow K_{\infty} \circ T_{\omega}$. Hence, we obtain that $K_{n}^{1}$ satisfies the same functional equation and the same asymptotics than $K_{n+1} \circ T_{\omega}$, so that

$$
K_{n}^{1}=K_{n+1} \circ T_{\omega} .
$$

Hence, using (27) we obtain that setting

$$
K_{n+a}=K_{n}^{a} \circ T_{-a \omega}
$$

it is a consistent definition. From equality (26), we obtain that with this previous definition (28),

$$
V_{n+a}^{n+a+b} \circ K_{n}^{a}=K_{n}^{a+b}=K_{n+a+b} \circ T_{\omega(a+b)},
$$

and also

$$
V_{n+a}^{n+a+b} \circ K_{n}^{a}=V_{n+a}^{n+a+b} \circ K_{n+a} \circ T_{\omega a} .
$$

Hence, we have the invariance equation

$$
V_{n+a}^{n+a+b} \circ K_{n+a}=K_{n+a+b} \circ T_{\omega b} .
$$

In other words, writing $t=n+a$, we obtain that definition (28) satisfies the invariance equation for all times. 
4.5. Smooth dependence on parameters. The proof of Theorem 3.1 using the Implicit Function Theorem as well as the differentiability properties in Lemma 4.2 has the following immediate corollary.

Corollary 4.7. Let $K_{\infty}$ be an (autonomous) invariant torus for $f_{\infty}$. Assume $\lambda \mu^{r}<1$. Then, the mapping

$$
\mathcal{T}: \mathcal{F}_{\lambda, r+l}^{0} \rightarrow \mathcal{K}_{\lambda, r}
$$

that associates to a sequence $\underline{f}$, the sequence $\underline{K}$ satisfying

$$
\mathcal{T}(\underline{f})=\underline{f} \circ \underline{K}-\underline{K} \circ T_{\omega}
$$

is $C^{l}$.

Note also that we have concrete formulas for the terms of map $\mathcal{T}$. Following the Implicit Function Theorem, we obtain that

$$
\mathrm{D} \mathcal{T}(\underline{f}) \eta=-\left(\mathrm{D}_{2} \mathcal{J}(\underline{f}, \underline{K})\right)^{-1} \Delta .
$$

Hence, if $f_{n}^{\varepsilon}=f_{n}^{0}+\varepsilon f_{1}+O^{2}$, we obtain that $K_{n}^{1}=K_{0}^{0}+\varepsilon K_{1}$.

\section{WHISKERED KAM TORI}

Consider again $f_{n}$ as sequence of mappings in such a way that

$$
f_{n} \rightarrow f_{\infty}
$$

Consider a time dependent KAM tori, that is a sequence $K_{n}: \mathbb{T}^{d} \rightarrow M$ such that

$$
f_{n} \circ K_{n}(\theta)=K_{n+1}(\theta+\omega) .
$$

For simplicity, in this paper we will only consider whiskered tori and its stable manifolds with one dimensional manifolds:

$$
W_{n}(\theta, 0)=K_{n}(\theta) \text {. }
$$

Definition 5.1. We will say that a time dependent whiskered torus is a sequence of mappings $W_{n}: \mathbb{T}^{d} \times I \rightarrow M$ such that satisfies

$$
f_{n} \circ W_{n}(\theta, s)=W_{n+1} \circ R_{\omega, \gamma}(\theta, s),
$$

where $R_{\omega, \gamma}(\theta, s)=(\theta+\omega, \gamma s)$.

In analogy with the case of KAM tori, we will supplement the invariance equation (29) with the asymptotic condition

$$
W_{n} \rightarrow W_{\infty},
$$

so then, if (30) is satisfied, we will have

$$
f_{\infty} \circ W_{\infty}(\theta, s)=W_{\infty} \circ R_{\omega, \gamma}(\theta, s),
$$


and $W_{\infty}$ will give a parameterization of a Whiskered KAM torus for the autonomous diffeomorphism. Analogous to remark 2.3, once we choose a parameterization of the autonomous whiskered KAM torus, the non-autonomous invariant whiskered torus is uniquely determined.

We will prove Theorem 5.2 which plays the same role for whiskered tori as Theorem 3.1 for tori.

Theorem 5.2 (Case of maps). Let $M$ be a manifold. Let $f_{n}: M \rightarrow M$ be a sequence of diffeomorphisms such that $\left\|f_{n}-f_{\infty}\right\|_{C^{r+2}} \leq \lambda^{n} C_{1}$, for some $\lambda \leq 1$ and $C_{1}>0$. Assume that $f_{\infty}$ has a whiskered KAM torus, that is, there exists a map $W_{\infty}: \mathbb{T}^{d} \times I \rightarrow M$ such that

$$
f_{\infty} \circ W_{\infty}(\theta, s)=K_{\infty}(\theta+\omega, \gamma s) .
$$

Assume that for some $\mu>0$ and $C>0$ we have:

(I) $\left\|\mathrm{D} f_{\infty} \circ W_{\infty}(\theta+n \omega, \gamma s) \cdots \mathrm{D} f_{\infty} \circ W_{\infty}(\theta, s)\right\|_{L^{\infty}} \leq(\gamma \mu)^{n} \tilde{C}$,

(II) $(\gamma \mu)^{r} \lambda<1$.

(III) $C_{1}$ sufficiently small.

Then, there exists a sequence of mappings $W_{n}: \mathbb{T}^{d} \times I \rightarrow M$ satisfying (29) and

$$
\left\|W_{n}-W_{\infty}\right\|_{C^{r}} \leq C_{* * *}(\lambda \gamma)^{n} .
$$

Furthermore, we can take $C_{* * *}=L C_{1}$, where $L$ is a constant depending only on the sequence $f_{n}$.

The sequence satisfying (29) and (31) is, with $C_{* * *} \leq C_{+}$(where $C_{+}$depends only on $f_{n}$ ) unique.

Proof. The proof will follow the same skeleton as the proof of the result of invariant tori.

We define first affine spaces of sequences of mappings $\mathbb{T}^{d} \times I \rightarrow M$.

Definition 5.3. We define a space of sequences of mappings on $\mathbb{T}^{d} \times I \rightarrow M$ as

$$
\mathcal{W}_{\lambda, \gamma, r}=\left\{\left\{W_{n}\right\}_{n=0}^{\infty} \mid\left\|W_{n}-W_{\infty}\right\|_{C^{r}} \leq C(\lambda \gamma)^{n}\right\} .
$$

And of course, we define for the sequences $\underline{W}, \tilde{W} \in \mathcal{W}_{\lambda, \gamma, r}$ the norm:

$$
\|\underline{W}-\tilde{W}\|_{\lambda, \gamma, r}=\sup _{n}\left\|W_{n}-W_{\infty}\right\|_{C^{r}}(\lambda \gamma)^{-n} .
$$

Note that $\mathcal{W}_{\lambda, \gamma, r}$ is an affine space modeled on the Banach space

$$
\mathcal{W}_{\lambda, \gamma, r}^{0}=\left\{\left\{\hat{W}_{n}\right\}_{n=0}^{\infty} \mid\left\|\hat{W}_{n}\right\|_{C^{r}} \leq C(\lambda \gamma)^{n} \text {, with }\left\|\hat{W}_{n}\right\|_{\lambda, \gamma, r}=\sup \left\|\hat{W}_{n}\right\|_{C^{r}}(\lambda \gamma)^{-n}\right\} \text {. }
$$

Proceeding in an analogous way as on the proof of Theorem 3.1, we can define the functional $\mathcal{Z}$ defined by

$$
\mathcal{Z}: \mathcal{F}_{\lambda, r+l+2} \times \mathcal{W}_{\lambda, \gamma, r} \rightarrow \mathcal{W}_{\lambda, \gamma, r}^{0}
$$


which is a $C^{l}$ function by Lemma 4.2. Assuming that $0<\lambda<1, r \in \mathbb{N}$, $l \in \mathbb{N}$ and $l \geq 1$, the derivative with respect to the second variable of $\mathcal{Z}$ is defined by

$$
\left(\mathrm{D}_{2} \mathcal{Z}(\underline{f}, \underline{W}) \Delta\right)_{n}:=\mathrm{D} f_{n} \circ W_{n} \Delta_{n}-\Delta_{n+1} \circ R_{\omega, \gamma} .
$$

Moreover, the derivative

$$
\mathrm{D}_{2} \mathcal{Z}\left(\underline{f}_{\infty}, \underline{W}_{\infty}\right): \mathcal{F}_{\lambda, r}^{0} \times \mathcal{W}_{\lambda, \gamma, r}^{0} \rightarrow \mathcal{W}_{\lambda, \gamma, r}^{0}
$$

is boundedly invertible under the rate conditions (I) and (II) of Theorem 5.2.

We need to show that given $\underline{\eta} \in \mathcal{W}_{\lambda, \gamma, r}^{0}$, we can find a unique $\Delta \in \mathcal{W}_{\lambda, \gamma, r}^{0}$ solving

$$
\mathrm{D} f_{\infty} \circ W_{\infty} \Delta_{n}-\Delta_{n+1} \circ R_{\omega, \gamma}=\eta_{n}
$$

and that

$$
\|\Delta\|_{\lambda, \gamma, r} \leq C\|\underline{\eta}\|_{\lambda, \gamma, r}
$$

Then, we can rewrite equation (32) as

$$
\Delta_{n}=M \Delta_{n+1} \circ R_{\omega, \gamma}+M \eta_{n}
$$

where $M=\left(\mathrm{d} f_{\infty} \circ W_{\infty}\right)^{-1}$. Repeating this process we are lead to

$$
\begin{aligned}
\Delta_{n}= & \sum_{j=0}^{L} M \circ R_{\omega, \gamma}^{j} \cdots M \eta_{n+j} \circ R_{\omega, \gamma}^{j} \\
& +M \circ R_{\omega, \gamma}^{L+1} \cdots M \Delta_{n+L+1} \circ R_{\omega, \gamma}^{L+1},
\end{aligned}
$$

where formally, Equation (33) suggests that we should have

$$
\Delta_{n}(\theta, s)=\sum_{j=0}^{\infty} M \circ R_{\omega \gamma}^{j}(\theta, s) \cdots M(\theta, s) \eta_{n+j} \circ R_{\omega, \gamma}^{j}(\theta, s) .
$$

The argument to justify (34) and to establish uniqueness is identical to the argument we use to justify the corresponding equations in Theorem 3.1.

For the whiskered tori, to see that the sum in (34) converges and gives a solution of (32), we have to take into account also the powers of $\gamma$, related to the contraction along the whiskers.

We can express the assumption (I) of Theorem 5.2, as

$$
\left|M \circ R_{\omega, \gamma}^{j}(\theta, s) \cdots M(\theta, s)\right| \leq \tilde{c}_{0} \mu^{j} \gamma^{j},
$$

satisfied for some $\tilde{c}_{0}>0$, and we consider the derivative (notice that now it depends on two variables) approximated by $\mathrm{D}_{s}^{l} \mathrm{D}_{\theta}^{k} R_{\omega, \gamma}^{j} \simeq \gamma^{l}$. Hence, we obtain:

$$
\left\|\mathrm{D}_{s}^{r} \mathrm{D}_{\theta}^{k}\left(M \circ R_{\omega, \gamma}^{j} \cdots M(\theta, s)\right)\right\| \leq \tilde{c}_{0}^{r}\|M\|_{C^{r}}^{r} j(j+1) \cdots(j+r) \mu^{r j} \gamma^{r j} .
$$


From the bounds of Equation (35) and the definition of $\mathcal{W}_{\lambda, r}^{0}$, we obtain that, if there is a solution $\Delta$ of Equation (33) in $\mathcal{W}_{\lambda, \gamma, r}^{0}$, the last term goes to 0 in $C^{r}$. Hence, we should have

$$
\begin{array}{rl}
\Delta_{n}=\lim _{L \rightarrow \infty} \sum_{j=0}^{L} & M \circ R_{\omega, \gamma}^{j} \cdots M \eta_{n+j} \circ R_{\omega, \gamma}^{j} \\
& +M \circ R_{\omega, \gamma}^{L+1} \cdots M \Delta_{n+L+1} \circ R_{\omega, \gamma}^{L+1},
\end{array}
$$

which limit is understood in $C^{r}$. So, we know that the solution $\Delta_{n}$ given in equation (34) is the only possible solution.

Now that we know that Equation (34) is the only possible solution, we investigate its properties and show that, indeed, is a solution and satisfies the desired bounds. Using Equation (35), we can bound the general term of Equation (34) by:

$$
\begin{aligned}
\left\|M \circ R_{\omega, \gamma}^{j} \cdots M \eta_{n+j} \circ R_{\omega, \gamma}^{j}\right\|_{C^{r}} & \leq\left\|M \circ R_{\omega, \gamma}^{j} \cdots M\right\|_{C^{r}}\left\|\eta_{n+j}\right\|_{C^{r}} \\
& \leq c_{0}^{r}\|M\|_{C^{r}}^{r} j(j+1) \cdots(j+r) \mu^{r j} \gamma^{r j}(\lambda \gamma)^{n+j}\|\eta\|_{\lambda, \gamma, r} \\
& =(\lambda \gamma)^{n}\|\eta\|_{\lambda, \gamma, r} c_{0}^{r}\|M\|_{C^{r}}^{r} \frac{(j+r) !}{j-1}\left(\mu^{r} \gamma^{r} \lambda\right)^{j} .
\end{aligned}
$$

Therefore, the series of Equation (34) converges uniformly, since $\mu^{r} \gamma^{r} \lambda<1$ by hypothesis. Hence, it is not difficult to justify that it is a solution by rearranging terms of series. We finally observe that

$$
\begin{aligned}
\left\|\Delta_{n}\right\|_{C^{r}} & \leq \lim _{L \rightarrow \infty} \sum_{j=0}^{L}(\lambda \gamma)^{n}\|\eta\|_{\lambda, \gamma, r} c_{0}^{r}\|M\|_{C^{r}}^{r} \frac{(j+r) !}{j-1}\left(\mu^{r} \gamma^{r} \lambda\right)^{j} \\
& \leq C(\lambda \gamma)^{n}\|\eta\|_{\lambda, \gamma, r} \leq C_{* * *}(\lambda \gamma)^{n}
\end{aligned}
$$

where $C_{* * *}$ depends only on the sequence $f_{n}$.

\section{GeOMETRIC PROPERTIES}

Note that in the discussion so far, we have only used regularity properties of the dynamical system and we have not used that the system preserves any geometric property.

In many applications, such as the ones presented in the introduction, the dynamical system are Hamiltonian or present other geometric properties. Such properties lead to many practical consequences such as efficient perturbation theory, etc.

The goal of this section is to present some arguments that show that, if the dynamical systems are Hamiltonian, we can obtain easily good geometric properties of the invariant tori. We will show that the time dependent KAM tori and the stable manifolds of the whiskers are Lagrangian manifolds (ie: the symplectic form vanishes on them). 
Lets recall first some basic definitions for autonomous systems.

Definition 6.1. A diffeomorphism $f: M \rightarrow M$ is called symplectic if it satisfies

$$
f^{*} \Omega=\Omega,
$$

where $\Omega$ is the symplectic form, and the pull-back form $f^{*}$ is defined on the tangent space $T M$.

Definition 6.2. Assume that $f: M \rightarrow M$ is symplectic. Then, $f$ is exact symplectic if there exists $S: M \rightarrow \mathbb{R}$ (the primitive function of $f$ ) such that

$$
\mathrm{d} S=f^{*} \alpha-\alpha
$$

where $\alpha$ is the action form of $\Omega$. In such a case, $\Omega=\mathrm{d} \alpha$ is the exact symplectic form.

Given a parameterization $K: \mathbb{T}^{d} \rightarrow M$ of the torus, the geometric structure on $M$ pulls back to the corresponding structure on $K\left(\mathbb{T}^{d}\right)$ via $K^{*} \Omega$. We say that a torus parameterized by $K$ is isotropic, if it satisfies:

$$
K^{*} \Omega=0 .
$$

Moreover, the invariant tori of maximal dimension are Lagrangian. Analogously, we say that a whiskered torus parameterized by $W$ is Lagrangian, if it satisfies:

$$
W^{*} \Omega=0 .
$$

It is well known that, if $f$ is exact symplectic and $K$ is the parameterization of an autonomous invariant torus for an irrational rotation, that is that it satisfies the invariance equation

$$
f \circ K=K \circ T_{\omega},
$$

then, the torus is isotropic (analogously for $W$ ) [Zeh75, Zeh76, dlLGJV05]. Of course, in an integrable system, even rational tori are isotropic.

Proposition 6.3. With the notation of Theorem 3.1 and 5.2, if $f_{n}$ is symplectic, then $K_{\infty}$ (respectively $W_{\infty}$ ) are isotropic, and so are the $K_{n}, W_{n}$ produced by Theorems 3.1 and 5.2. In particular, if they are of maximal dimension, they are Lagrangian.

Proof. By Theorem 3.1, we know that if $f_{\infty}$ has a (autonomous) KAM torus parameterized by $K_{\infty}$, then there exists a sequence of embeddings $K_{n}$ satisfying

$$
f_{n} \circ K_{n}=K_{n+1} \circ T_{\omega},
$$

and also, that this sequence converges sufficiently fast to $K_{\infty}$. By applying the pull-back to the invariance equation (36) we obtain

$$
K_{n}^{*} \circ f_{n}^{*} \Omega=T_{\omega}^{*} \circ K_{n+1}^{*} \Omega .
$$


Assume now that $f_{n}$ is symplectic, that is $f_{n}^{*} \Omega=\Omega$, then, we want to see that $K_{n}^{*} \Omega=0$. If $f_{n}$ is symplectic, the previous equation (37) is just

$$
K_{n}^{*} \Omega=T_{\omega}^{*} K_{n+1}^{*} \Omega .
$$

If $f_{n}$ symplectic, also $f_{n+1}^{*} \Omega=\Omega$, so we can rewrite equation (38) as

$$
K_{n}^{*} \Omega=T_{\omega}^{*} K_{n+1}^{*} f_{n+1}^{*} \Omega .
$$

By the invariance on $K_{n}$, we have $f_{n+1} \circ K_{n+1}=K_{n+2} \circ T_{\omega}$ and

$$
K_{n}^{*} \Omega=T_{\omega}^{*}\left(K_{n+2} \circ T_{\omega}\right)^{*} \Omega,
$$

that is

$$
K_{n}^{*} \Omega=\left(T_{\omega}^{*}\right)^{2} K_{n+2}^{*} \Omega .
$$

Repeating the previous process $l$ times, we obtain

$$
K_{n}^{*} \Omega=\left(T_{\omega}^{*}\right)^{l} K_{n+l}^{*} \Omega,
$$

which can be bounded as

$$
\left\|K_{n}^{*} \Omega\right\|_{C^{0}}=\left\|\left(T_{\omega}^{*}\right)^{l} K_{n+l}^{*} \Omega\right\|_{C^{0}} \leq\left\|K_{n+l}^{*} \Omega\right\|_{C^{0}} .
$$

Then, by using that $K_{\infty}$ is isotropic, that is $K_{\infty}^{*} \Omega=0$, and that $K_{n} \rightarrow K_{\infty}$ (so that $K_{n+l} \rightarrow K_{\infty}$ ), we obtain that $K_{n+l}^{*} \Omega \rightarrow 0$. That is, the non-autonomous KAM tori given by the sequence $K_{n}$ is isotropic.

Using the same arguments, we obtain that $W_{n+l}^{*} \rightarrow 0$, so that $W_{n}$ is isotropic.

\section{NON-EUCLIDEAN MANIFOLDS}

In the case that $M$ is not an Euclidean manifold one can take reformulate the problem in terms of sections of vectors. Very similar discussions happen in [Mos69], [dlLMM86, Appendix A].

By the tubular neighborhood theorem, [AR67], we have a system of coordinates in a neighborhood of the torus invariant for the autonomous system. We can assume that the system of coordinates is $C^{\infty}$ by substituting $K_{\infty}$ by a $C^{\infty}$ approximation.

More precisely, we can assume that there is a $C^{\infty}$ metric and we can write any point $p$ in a sufficiently small neighborhood of a torus in a unique way as $p=\exp _{s}(v)$, where $s$ is a point in the torus and $v$ is orthogonal to the tangent space and sufficiently small. So, a point $p$ can be associated to the coordinates $s, v$ as above. Then, a torus in this neighborhood can be associated to a section of the normal bundle close to the zero section if the point $s$ is given by $s=K_{\infty}(\theta)$.

Similarly, the differences between two mappings can be written using the exponential mapping. We associate to $f_{n}-f_{\infty}$ the vector field $v_{n}$ such that $\exp _{f_{\infty}(x)}\left(v\left(f_{\infty}(x)\right)=f_{n}(x)\right.$. Also, we can identify $K_{n}-K_{\infty}$ as another vector field defined in the image of $K_{\infty}$. 
The equation (6) can be expressed as an equation for maps. The main observation is that since the composition of near identity maps corresponds to an operation which is very similar to the addition of the vector fields, the computations we have carried out, in particular, the Implicit Function Theorem, carry out directly.

Note that with our formulation (which is the same as [dlLMM86, Appendix A]) the operator is differentiable and we can get differentiability with respect to parameters. For Anosov systems (for which $K$ is a diffeomorphism) [Mos69] writes a different - but equivalent - giving the solutions as the zeros of another operator.

\section{AcKNOWLEDGMENTS}

M.C. and R.L. have been supported by NSF DMS-1500943. M.C. also supported by MTM2012-32541. We thank T. Bartsch, D. Blazevski and A. Haro for encouraging us.

\section{APPENDix A. Summary of THE GLObal SCATTERING THEORY For TIME} DEPENDENT CLASSICAL SYSTEMS

Let $X_{t}, Y_{t}$ be two vector fields. We denote by $V_{t_{0}}^{t}, U_{t}$ the evolution of $X_{t}$. That is

$$
\begin{aligned}
& \frac{d}{d t} V_{t_{0}}^{t}=X_{t} \circ V_{t_{0}}^{t}, V_{t_{0}}^{t_{0}}=I d, \\
& \frac{d}{d t} U_{t}=Y \circ U_{t}, U_{0}=I d .
\end{aligned}
$$

Assume that $X_{t} \rightarrow Y$ sufficiently fast (in a $C^{r}$ norm).

Inspired by the quantum mechanical scattering theory [BdlL11, Hei43b, Hei43a, Whe37], we define

$$
\Omega_{ \pm, t_{0}}=\lim _{t \rightarrow \pm \infty} U_{t-t_{0}} \circ V_{t_{0}}^{t},
$$

where $\Omega_{ \pm, t_{0}}(x)$ gives the initial point of the trajectories under $Y$, which is asymptotic (in the future, respectively in the past) to the trajectories of $X_{t}$ starting at $x$ at time $t_{0}$. The paper [BdlL11] shows that if for some $r \in \mathbb{N}$

$$
\left\|X_{t}-Y\right\|_{C^{r}} \leq \theta^{t} C
$$

with $\theta \leq \theta_{0}(r)<1$, and $\theta_{0}(r)$ an explicit funtion of the Lyapunov exponents of $Y$, then, the limits in $C^{r-1}$ of (39) exists and are diffeomorphisms.

Note that it is clear that if $V_{t}$ preserve any geometric structure (e.g. symplectic, presymplectic, Poison), so does the limit in (39). It is also clear that if the limits in (39) exist, we also have

$$
\Omega_{ \pm, t_{0}} \circ V_{t_{0}-\delta}^{t_{0}}=U_{\delta} \circ \Omega_{ \pm, t_{0}-\delta} .
$$


When $\Omega_{ \pm}$is a diffeomorphism, we have

$$
V_{t_{0}-\delta}^{t_{0}}=\Omega_{ \pm, t_{0}}^{-1} \circ U_{\delta} \circ \Omega_{ \pm, t_{0}-\delta} .
$$

In particular, if $\Psi$ is an object (e.g. a manifold or a Cantor set, etc.) invariant under $U_{\delta}$, then it satisfies

$$
V_{t_{0}-\delta}^{t_{0}}\left(\Psi_{t_{0}-\delta}\right)=\left(\Psi_{t_{0}}\right)
$$

where $\Psi_{t_{0}-\delta} \equiv \Omega_{ \pm, t_{0}-\delta}^{-1} \Psi$, so that the family $\Psi_{t_{0}}$ gives an invariant object for the autonomous system.

We can consider $\Omega_{ \pm, t_{0}}^{-1}$ as a time dependent embedding of the reference object $\Psi$ into the phase space. When we express the dynamics on $\Psi_{t}$ into the coordinates of $\Psi$, we obtain that it is given by $V_{t}$, and the dynamics

$$
\Omega_{ \pm, t_{0}} \circ U_{t_{0}-\delta}^{t_{0}} \circ \Omega_{ \pm, t_{0}-\delta}^{-1}=V_{\delta} .
$$

Note that the above calculations apply not only when $\Psi$ is a manifold, but could also be a horseshoe or any other object.

The global scattering theory of [BdlL11] gives not only the embeddings of an invariant object, but also the fact that these objects fit together. For example, if $\Psi^{\omega}$ is a Cantor family of KAM tori parameterized by the frequency $\omega$ over a Cantor set and depending $C^{\infty}$-Whitney on $\omega$, the time dependent counterparts $\Psi_{t}^{\omega}$ will also be a $C^{\infty}$-Whitney differentiable family. Numerical algorithms and implementations in some famous examples can be found in $[\mathrm{BF} 12]$.

\section{REFERENCES}

[ALF05] S.A. Astakhov, E.A. Lee, and D. Farrelly. Formation of kuiper-belt binaries through multiple chaotic scattering encounters with low-mass intruders. Mon. Not. R. Astron. Soc., (360):401-415, 2005.

[AR67] R. Abraham and J. Robbin. Transversal mappings and flows. An appendix by Al Kelley. W. A. Benjamin, Inc., New York-Amsterdam, 1967.

[BdlL11] D. Blazevski and R. de la Llave. Time-dependent scattering theory for ODEs and applications to reaction dynamics. J. Phys. A, 44(19):195101, 26, 2011.

[BF12] D. Blazevski and J.K. Franklin. Using scattering theory to compute invariant manifolds and numerical results for the laser-driven hnon-heiles system. Chaos, 22:043138, 2012.

$\left[\mathrm{BMH}^{+} 08\right]$ T. Bartsch, J. M. Moix, R. Hernandez, S. Kawai, and T. Uzer. Timedependent transition state theory. Adv. Chem. Phys., 140:191-238, 2008.

[BRBB12] T. Bartsch, F. Revuelta, R. M. Benito, and F. Borondo. Reaction rate calculation with time-dependent invariant manifolds. J. Chem. Phys., 136:224510, 2012.

[BUMH08] T. Bartsch, T. Uzer, J. M. Moix, and R Hernandez. Transition state theory rate calculations with a recrossing-free moving dividing surface. J. Phys. Chem. B, 112:206-212, 2008. 
[CV02] V. V. Chepyzhov and M. I. Vishik. Attractors for equations of mathematical physics, volume 49 of American Mathematical Society Colloquium Publications. American Mathematical Society, Providence, RI, 2002.

[dCNGM96] D. del Castillo-Negrete, J. M. Greene, and P. J. Morrison. Area preserving nontwist maps: periodic orbits and transition to chaos. Phys. D, 91(1-2):123, 1996.

[Die69] J. Dieudonné. Foundations of modern analysis. Academic Press, New York-London, 1969. Enlarged and corrected printing, Pure and Applied Mathematics, Vol. 10-I.

[dlLGJV05] R. de la Llave, A. González, À. Jorba, and J. Villanueva. KAM theory without action-angle variables. Nonlinearity, 18(2):855-895, 2005.

[dlLMM86] R. de la Llave, J. M. Marco, and R. Moriyón. Canonical perturbation theory of Anosov systems and regularity results for the Livšic cohomology equation. Ann. of Math. (2), 123(3):537-611, 1986.

[dlLO99] R. de la Llave and R. Obaya. Regularity of the composition operator in spaces of Hölder functions. Discrete Contin. Dynam. Systems, 5(1):157184, 1999.

[Eas91] R. W. Easton. Capture orbits and Mel'nikov integrals in the planar threebody problem. Celestial Mech. Dynam. Astronom., 50(3):283-297, 1991.

[FdILS09a] E. Fontich, R. de la Llave, and Y. Sire. Construction of invariant whiskered tori by a parameterization method. I. Maps and flows in finite dimensions. J. Differential Equations, 246(8):3136-3213, 2009.

[FdlLS09b] E. Fontich, R. de la Llave, and Y. Sire. A method for the study of whiskered quasi-periodic and almost-periodic solutions in finite and infinite dimensional Hamiltonian systems. Electron. Res. Announc. Math. Sci., 16:9-22, 2009.

[FW14] A. Fortunati and S. Wiggins. Persistence of Diophantine flows for quadratic nearly integrable Hamiltonians under slowly decaying aperiodic time dependence. Regul. Chaotic Dyn., 19(5):586-600, 2014.

[Hal80] Jack K. Hale. Ordinary differential equations. Robert E. Krieger Publishing Co., Inc., Huntington, N.Y., second edition, 1980.

[Hal88] J. K. Hale. Asymptotic behavior of dissipative systems, volume 25 of Mathematical Surveys and Monographs. American Mathematical Society, Providence, RI, 1988.

[Har02] Philip Hartman. Ordinary differential equations, volume 38 of Classics in Applied Mathematics. Society for Industrial and Applied Mathematics (SIAM), Philadelphia, PA, 2002. Corrected reprint of the second (1982) edition [Birkhäuser, Boston, MA; MR0658490 (83e:34002)], With a foreword by Peter Bates.

[HdILS12] G. Huguet, R. de la Llave, and Y. Sire. Computation of whiskered invariant tori and their associated manifolds: new fast algorithms. Discrete Contin. Dyn. Syst., 32(4):1309-1353, 2012.

[HdlLS13] G. Huguet, R. de la Llave, and Y. Sire. Fast iteration of cocycles over rotations and computation of hyperbolic bundles. Discrete Contin. Dyn. Syst. $S$, pages 323-333, 2013. Issue special.

[Hei43a] W. Heisenberg. Die beobachtberen Grossen in der Theorie der Elementarteilch en II. Z. Phys., 120:673-702, 1943. 
[Hei43b] W. Heisenberg. Die beobachtberen Grossen in der Theorie der Elementarteilchen. Z. Phys., 120:3-46, 1943.

[Irw72] M. C. Irwin. On the smoothness of the composition map. Quart. J. Math. Oxford Ser. (2), 23:113-133, 1972.

$\left[\mathrm{KBJ}^{+} 07\right]$ S. Kawai, A. D. Bandrauk, C. Jaffé, T. Bartsch, J. Palacián, and T. Uzer. Transition state theory for laser-driven reactions. J. Chem. Phys., 126:164306, 2007.

[KR11] P. E. Kloeden and M. Rasmussen. Nonautonomous dynamical systems, volume 176 of Mathematical Surveys and Monographs. American Mathematical Society, Providence, RI, 2011.

[LAAF07] E. Lee, S. A. A. Astakhov, and D. Farrelly. Production of transneptunian binaries through chaos-assisted capture. Mon. Not. R. Astron. Soc., 379:229-246, 2007.

[MEdCCR10] C. G. L. Martins, R. Egydio de Carvalho, I. L. Caldas, and M. Roberto. The non-twist standard map with robust tori. J. Phys. A, 43(17):175501, 8, 2010.

[Mor00] P. J. Morrison. Magnetic field lines, Hamiltonian dynamics, and nontwist systems. Phys. Plasmas, 7(6):2279-2289, 2000.

[Mos69] J. Moser. On a theorem of Anosov. J. Differential Equations, 5:411-440, 1969.

[Sch69] J. T. Schwartz. Nonlinear functional analysis. Gordon and Breach Science Publishers, New York-London-Paris, 1969. Notes by H. Fattorini, R. Nirenberg and H. Porta, with an additional chapter by Hermann Karcher, Notes on Mathematics and its Applications.

[SHF10] A. G. Suarez, D. Hestroffer, and D. Farrelly. Formation of the extreme kuiper-belt binary $2001 q w_{322}$ through adiabatic switching of orbital elements. Celest. Mech. Dyn. Astron., 106:245259, 2010.

[VZC13] M. I. Vishik, S. V. Zelik, and V. V. Chepyzhov. Regular attractors and their nonautonomous perturbations. Mat. Sb., 204(1):3-46, 2013.

[Whe37] J. A. Wheeler. On the mathematical description of light nuclei by the method. of resonating group structure. Phys. Rev., 52:11071122, 1937.

[Zeh75] E. Zehnder. Generalized implicit function theorems with applications to some small divisor problems. I. Comm. Pure Appl. Math., 28:91-140, 1975.

[Zeh76] E. Zehnder. Generalized implicit function theorems with applications to some small divisor problems. II. Comm. Pure Appl. Math., 29(1):49-111, 1976.

School of Mathematics, Georgia Institute of Technology, 686 Cherry Street, AtLANTA GA 30332

E-mail address: marta.canadell@math.gatech.edu

School of Mathematics, Georgia Institute of Technology, 686 Cherry Street, AtLANTA GA 30332

E-mail address: rafael.delallave@math.gatech.edu 